\title{
Energieberatung in Haushalten auf Basis des Smartmetering
}

\author{
Stromeinsparungen sind ein zentraler Aspekt \\ einer nachhaltigen Energienutzung. Ein software- \\ basierter Beratungsansatz könnte helfen, den \\ Umgang mit elektrischer Energie bewusster zu \\ machen und so zu einem angepassten Strom- \\ konsum beitragen. \\ Von Thorsten Raabe, Michael Sonnenschein, \\ Petra Beenken, Andreas Hüwel und \\ Christopher-Marcel Meinecke
}

M it dem Schlagwort „Energiewende“ ist die Zukunft einer Stromversorgung auf Basis regenerativer Energien nur sehr unvollständig beschrieben. Bereits absehbare Konsequenzen für Versorgungssysteme und Stromkonsumenten begründen Herausforderungen, die es gilt kritisch und konstruktiv zu evaluieren. Ein weiterer Ausbau der Stromnetze erscheint unumgänglich (Dena 2010). Strom wird in erheblichem Umfang in dezentralen Kleinkraftwerken erzeugt werden. Die Steuerung eines solchen Pools von Kraftwerken mit dem Ziel einer Deckung des momentanen Verbrauchs ist eine Herausforderung, der durch das Konzept virtueller Kraftwerke begegnet werden soll (Bitsch et al. 2002). Haushalte als Stromverbraucher werden aufgrund der zeitlich schwankenden Einspeisungen aus Erneuerbaren Energien verstärkt in die Stabilisierung der Stromversorgung integriert werden müssen. Das Paradigma „Erzeugung folgt Last“ wird sich zumindest teilweise umkehren zu „Last folgt Erzeugung“. Einschlägige Maßnahmen werden unter dem Begriff „Demand Side Management“ thematisiert (Sonnenschein et al. 2010).

Für Haushalte als Stromkonsumenten verbinden sich unter dem Stichwort „adaptive households“ mit den skizzierten Entwicklungen deshalb umfängliche Anpassungsleistungen. Es ist davon auszugehen, dass sich der bisher vielfach unreflektierte Umgang mit elektrischer Energie in Haushalten zugunsten eines reflektierten, „smarten“ Stromkonsums wandeln muss. Der Beitrag greift diese Entwicklungsperspektiven auf und liefert als Werkstattbericht den Zwischenstand der Ergebnisse eines Forschungsprojekts, das insbesondere die Konsequenzen für die Rolle privater Stromkonsumenten und deren Haushaltsführung im Rahmen der Umstellung des Stromversorgungssystems auf regenerative Energien untersucht (1). Ziel dieses (Teil-)Projektes ist es, in einem kombiniert sozialwissenschaftlich und informatik-basierten Zugang Perspektiven des Leitbilds „adaptiver Haushalte“ hinsichtlich seiner technischen wie auch verhaltens- und haushaltstheoretischen Konsequenzen zu präzisieren und in Form eines softwarebasierten Haushaltsberatungsansatzes zu konkretisieren.

\section{Nachhaltiger Stromkonsum als Ziel des Beratungsansatzes}

Die skizzierte Ausgangssituation wirft die Frage nach geeigneten und wirksamen Unterstützungsmöglichkeiten für die Stärkung aktiver Verbrauchskontroll- und Verbrauchsgestaltungsfähigkeit der Haushalte auf. Energieeinsparung und Adaption des Verbrauchs an volatile Stromeinspeisung stellen Ziele dar, die sowohl mit Effizienz-, Suffizienz- und Konsistenzpfaden der Entwicklung nachhaltigen Konsums verbunden sind. Der Ersatz von „Stromfressern“ durch energiesparsamere Haushaltsgeräte vermag die Ressourcenproduktivität im Haushalt zu erhöhen (Effizienz). Mit Änderung von Haushaltspraktiken beziehungsweise deren Reorganisation lassen sich ebenfalls Effizienzziele erreichen, wenn diese durch anderen oder geringeren Geräteeinsatz zur gleichen Nutzenstiftung führen. Dazu gehören beispielsweise eine geringere Waschtemperatur bei der Kleiderreinigung, die Nutzung alternativer Gartechniken wie Dampfdruckkochen oder die ausschließliche Nutzung maximal gefüllter Waschmaschinen. Die Reflektion der Notwendigkeit einzelner Haushaltspraktiken stellt eine Basis für Perspektiven suffizienten Konsumverhaltens dar. So ist durchaus denkbar und praktikabel, einzelne Gewohnheiten und Verrichtungen abzustellen beziehungsweise zu reduzieren und somit den Gesamtverbrauch des Haushaltes zu senken. Dazu gehören beispielsweise der Verzicht auf durchgängige Nutzung des TV-Geräts zur Hintergrundbeschallung, die Senkung der Zimmertemperatur oder die Substitution von energieintensiven Formen der Essenszubereitung. Mit dem Ziel der zeitlichen Verschiebung des Stromkonsums in Anpassung an Einspeisungsmengen, einem adaptiven Stromkonsum, sind unmittelbar (Natur-)konsistente Verhaltensmuster vorgezeichnet.

\section{Energieberatung in Haushalten}

Tatsächlich gibt es bereits eine Reihe von Energieberatungsangeboten, die insbesondere auf die Vermittlung von Informationen zu Energieeffizienz von Geräten ausgelegt sind. Dazu gehören die Angebote der DENA (2) oder $\mathrm{CO}_{2}$ online (3). Diese ausschließlich auf Informationsvermittlung setzenden Beratungsangebote stoßen jenseits des Austauschs von „Energiefressern“ und gerätebezogenen Verbrauchskontrollen allerdings an 
Grenzen. Tatsächlich ist der Stromkonsum in privaten Haushalten aufs Engste verknüpft mit der Lebensgestaltung seiner Mitglieder. Eine Vielzahl der Tätigkeiten im Haushalt sind als Routine organisiert, was den einzelnen Mitgliedern spezifische Entlastungen sichert. Daraus folgt, dass eine angestrebte Veränderung im Stromkonsum jenseits der energetischen Modernisierung der Geräteausstattung stets mit Veränderungen von häufig habitualisierten Haushaltsprozessen verbunden ist. Wichtigster Zugang zur Modifikation habitualisierter Verhaltensweisen ist die persönliche Aktivierung und Bewusstmachung der überwiegend unbewusst vollzogenen Handlungen (Dahlstrand/Biehl 1997). Für die Formulierung eines weitergehenden Beratungsansatzes ist deshalb zunächst zu schließen, dass die Energieberatung auf der Basis haushaltsindividueller Verbrauchsdaten vorgenommen werden sollte. So könnte auf Basis einer Erfassung des haushaltsspezifischen Stromkonsums über die Zeitachse eine Verbindung zu konkreten Tätigkeiten und Haushaltsprozessen hergestellt werden, deren Reflektion und Evaluation dann Ausgangspunkt für die durch die Beratung intendierte Verhaltensänderungen sein könnte.

\section{Technische Grundlagen einer lastgangbasierten Energieberatung}

Die technischen Voraussetzungen für diesen Beratungsansatz werden zurzeit durch die Installation von elektronischen Stromzählern, sogenannten Smart-Metern, Schritt für Schritt geschaffen. Als Ersatz für die herkömmlichen Stromzähler ermöglichen es Smart-Meter, den Verbrauch des individuellen Haushaltes in vergleichsweise hochaufgelöster Form über die Zeitachse zu erfassen und zu visualisieren. Ein Lastgang bezeichnet ganz grundsätzlich die in einer bestimmten Zeitperiode abgenommene elektrische Leistung (Müller 2010). Der Ge-

Abbildung 1: Lastgang eines Haushalts (Ausschnitt)

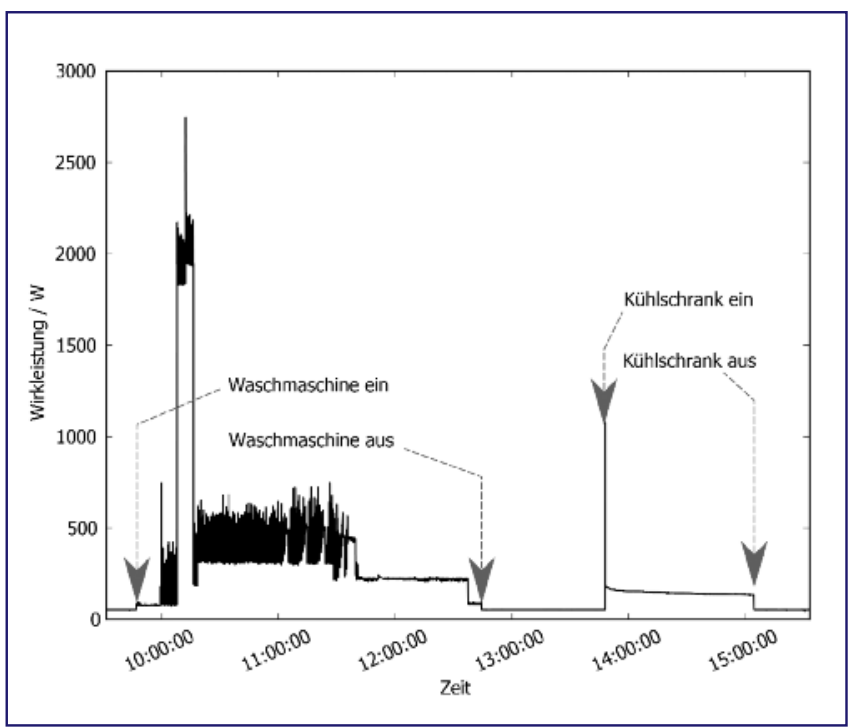

Quelle: Klingenberg 2010 samtlastgang stellt dabei für eine fest definierte Zeitperiode die Summe aller über einzelne Geräte entnommener elektrischer Leistung dar, während sich die gerätespezifischen Lastgänge aus der jeweils von einem spezifischen Haushaltsgerät entnommenen elektrischen Leistung für einen bestimmten Zeitraum ergeben.

Verschiedene Smart-Meter unterscheiden sich deutlich in der zeitlichen Auflösung, mit der die Gesamtlastgänge erhoben werden. Sie reichen von 15 Minuten bis zu 0,04 Sekunden. Seit mehr als 20 Jahren wird unter dem Begriff NIALM (Non-Intrusive-Appliance-Load-Monitoring) versucht, Gerätelastgänge aus einem gemessenen Gesamtlastgang zu extrahieren (Hart 1992). Verfahren wie etwa in Pihala (1998) oder Baranski (2006) beruhen darauf, Schaltereignisse von Geräten zu detektieren, indem Veränderungen in der gemessenen Wirk- und gegebenenfalls Blindleistung im Gesamtlastgang automatisch erkannt werden. Wie gut solche Schaltereignisse in einem Gesamtlastgang zu „sehen“ sind, hängt ganz wesentlich von der zeitlichen Auflösung der Messung des Lastgangs ab.

Unterschiedliche Geräte erzeugen verschiedene Leistungsveränderungen, mit denen sie grundsätzlich unterschieden werden können. Die automatische Zuordnung solcher Schaltereignisse zu Geräten beziehungsweise Gerätegruppen bedarf eines Klassifikationsverfahrens etwa auf der Grundlage künstlicher neuronaler Netze. Dies ist grundsätzlich möglich mit einer großen Datenbank der „Signaturen“ gängiger Geräte oder mit dem individuellen Training der Klassifikatoren in einem einzelnen Haus auf die dort eingesetzten Geräte. Letzteres kann vollautomatisch durch die Verwendung von Submetern erfolgen, die für die Trainingszeit der Klassifikatoren im Haushalt an den einzelnen Geräten installiert werden und parallel zu dem SmartMeter Schaltereignisse aufzeichnen.

Darauf aufbauend kann prinzipiell die Identifikation von Prozessen im Haushalt wie Mahlzeitzubereitung oder Wäschepflege erfolgen. Ein Prozess ist hier als eine Folge von Gerätenutzungen definiert. Eine automatische Prozesserkennung muss also dazu in der Lage sein, durchaus unterschiedliche Sequenzen von Gerätenutzungen als gleiche Prozesse zu identifizieren. Dies stellt aber insbesondere für den Fall zeitlich überlagerter Prozesse eine methodische Herausforderung dar, zu der zwar erste Ansätze existieren, die jedoch noch erheblicher Forschungsanstrengung bedarf.

\section{Ansatz einer lastgangbasierten Beratungssoftware}

Im Rahmen des Projektes „eTelligence“ wird ein „lastgangbasiertes Beratungssystem auf Softwarebasis“ entwickelt, dessen Grundidee auf zwei Merkmalen beruht.

Das erste Merkmal ist, dass die Beratung softwarebasiert, also ohne den Einbezug eines Energieberaters oder anderer zusätzlicher Beratungsleistungen, über den Heim-PC des beratenen Haushaltes erfolgt. Die Beratung ist damit durch ein hohes $\mathrm{Maß}$ an Kontinuität bei gleichzeitiger Autonomie geprägt. $\quad \rightarrow$ 
Sie kann immer dann stattfinden, wenn der Nutzer dies wünscht und unterliegt keiner zeitlichen Begrenzung.

Vor-Ort-Beratungen werden durch einen ins Haus kommenden Berater durchgeführt und zeichnen sich vor allem durch die Beratungsintensität und -tiefe aus. Diese Form der Beratung ist die „intensivste Form der persönlichen Stromsparberatung“ (Duscha et al. 2006). Der Nachteil ist, dass die Beratung an den ins Haus kommenden Berater gekoppelt ist und sie aufgrund der hohen Kosten nicht kontinuierlich, sondern nur punktuell und zeitlich beschränkt stattfinden kann. In der Folge können Einspareffekte, die auf der Basis von Verhaltens- und Nutzungsänderungen beruhen, nur bedingt erreicht werden. Während Empfehlungen, die sich auf Kaufentscheidungen, beispielsweise eine sparsamere Waschmaschine, und Kleininvestitionen wie den Kauf von Steckerleisten beziehen, sich bereits durch einmalige Handlungen umsetzen lassen, sind nutzungsbedingte Einspareffekte nicht so leicht zu erzielen. Die Nutzung elektronischer Geräte ist in die Alltagsgestaltung der Haushalte integriert und basiert auf automatisierten Routinen und Abläufen, die zu einem wesentlichen Teil unbewusst ablaufen. Um das auf der Handlungsebene liegende Einsparpotenzial - welches Bürger (2010) auf 20 Prozent des Gesamtverbrauchs eines Haushaltes schätzt - nutzen zu können, müssen diese Routinen und Abläufe hinterfragt und aufgebrochen und zugleich durch neue, stromsparende Handlungs- und Verhaltensweisen ersetzt werden.

Im Rahmen einer Vor-Ort-Beratung durch einen Berater ist dies kaum zu leisten, da das Hinterfragen, Aufbrechen und Ersetzen stromverbrauchender Verhaltensroutinen kontinuierlich, bestenfalls täglich über einen längeren Zeitraum erfolgen muss. Ansonsten besteht die Gefahr, dass die neu erlernten Verhaltensweisen im Sinne eines Fallback-Effekts sukzessiv wieder durch die alten Routinen ersetzt werden. Verschiedene Studien haben zur Vermeidung solcher Effekte und zur Erzielung langfristiger Einsparungen die Kontinuität von Feedback- und Beratungsleistung als entscheidendes Kriterium identifiziert (u.a. Arvola et al. 1993). Darby gibt als Daumenregel drei Monate oder länger an, die es dauert, neues Verhalten dauerhaft zu entwickeln und langfristige Einsparerfolge zu erzielen (Darby 2006).

Das zweite Merkmal des Beratungssystems ist, dass es nicht nur auf den zeitlich relativ hoch aufgelösten Gesamtverbrauchsdaten der Haushalte, sondern auch auf den daraus extrahierten Verbrauchsdaten einzelner Geräte beruht. Damit kann eine detaillierte Aufschlüsselung des Gesamtverbrauchs herausgearbeitet werden, auf dessen Basis eine möglichst individuelle Beratung hinsichtlich eines stromsparenden Verhaltens erfolgt.

Das System setzt sich damit von vielen anderen Smart-Meter-basierten Feedbacksystemen ab. Über Softwareprogramme, die auf dem Heim-PC installiert sind, über ortsungebundene Online-Portale oder über Anwendungen, sogenannte Applications, kurz: Apps, etwa für Smart-Phones, können die Verbraucher mit diesen Systemen ihren eigenen Haushaltsverbrauch zumeist über 15-Minuten-Daten kontrollieren. Sie zeichnen sich gegenüber der Vor-Ort-Beratung vor allem durch die Möglichkeit der kontinuierlichen Kontrolle und der zeitlichen Unabhängigkeit aus und sind im Zuge eines Smart-Meter-Rollouts für den flächendeckenden Einsatz geeignet.

Diesem Vorteil steht zugleich aber der Nachteil gegenüber, dass die Beratungstiefe und -intensität gering ist - sofern überhaupt von Beratung im eigentlichen Sinne gesprochen werden kann. Denn betrachtet wird in der Regel lediglich der Gesamtverbrauch des jeweiligen Haushaltes, während eine gerätespezifische Betrachtung und eine darauf basierende Reflektion einzelner Abläufe und stromnutzungsrelevanter Handlungen kaum stattfinden kann. Damit fällt eine Verknüpfung konkreter Handlungen mit dem aus ihnen jeweils resultierenden Stromverbrauch schwer, obwohl genau dieser Ansatz der anwendungsbezogenen Aufschlüsselung des Gesamtverbrauchs in verschiedenen Studien den größten Einsparerfolg erzielen konnte (Ueno et al. 2006). Zudem sind Hinweise auf den Austausch ineffizienter Geräte durch sparsamere kaum möglich. Erste Forschungsergebnisse zur Wirkung von Smart-Meter-basierten Feedbacksystemen, bei denen ein Einspareffekt von lediglich 3,7 Prozent festgestellt werden konnte, stützen diese vermutete Beschränkung hinsichtlich der Einsparwirkung (Schleich et al. 2011).

Während sich die Vor-Ort-Beratung also durch eine hohe Beratungstiefe auszeichnet und die Smart-Meter-basierten Feedbacksysteme durch die Möglichkeit der kontinuierlichen Kontrolle wirken, unterliegen sie zugleich jedoch im Falle der Vor-Ort-Beratung einer zeitlichen Beschränktheit oder im Falle der Feedback-Systeme einer geringen Beratungstiefe. Der hier skizzierte Ansatz eines „lastgangbasierten Beratungssystems auf Softwarebasis“ versucht nun, die Vorteile beider Ansätze in einem System zu integrieren und der Handlungsebene der Haushalte eine besondere Beachtung zu schenken.

\section{Stromeinsparung durch Konzentration auf Handlungsroutinen}

Es wird der Ansatz verfolgt, dass mit einer Beratung zur Gerätenutzung in Verbindung mit Handlungsroutinen und Verhaltensweisen ein größerer Effekt erzielt werden kann, als wenn eine isolierte Betrachtung der Gerätenutzung ohne diese Verbindung vorgenommen wird. Über die Erkennung von energienutzungsrelevanten Haushaltsprozessen wird für die Haushalte eine Art „identitätsstiftender Faktor“ in das Beratungssystem eingebracht. Konzeptionell meint dies, möglichst weitführende und auf den jeweiligen zu beratenden Haushalt hin individualisierte Verhaltensempfehlungen zu geben, durch die Verhaltensänderungen hin zu einem sparsameren Umgang mit Strom erreicht werden sollen. Dieses Ziel soll mit dem Beratungssystem über zwei grundlegende Komponenten erreicht werden.

Zunächst geschiet dies über eine informative Komponente. Strom ist ein besonderes Gut, denn es „hat kein Gewicht, kein Volumen und ist nicht sichtbar" (Brohmann et al. 2000) und der Mensch hat kein „Sinnesorgan zur direkten Wahrnehmung sei- 
nes Stromkonsums“ (Baranski 2006). Aufgabe des Systems ist es, das unsichtbare Gut Strom sichtbar und ansatzweise "greifbar" zu machen. Zudem geht es darum, zwischen den im Haushalt ablaufenden Handlungen und Prozessen und dem Verbrauch über die Nutzung der Geräte eine Verbindung herzustellen. Mit dem Konsum von Strom ist kein Selbstzweck verbunden. Der Konsum elektrischer Energie geht immer mit Aktivitäten und der Nutzung anderer Geräte einher, sodass es für den Konsumenten gar nicht so leicht ist, zu begreifen, welche Auswirkungen Stromsparen auf das tägliche Leben eigentlich hat (Fischer 2008).

Die auf der Informationskomponente aufsetzende Beratungskomponente hat die Aufgabe, die im Haushalt vorliegenden Routinen und Abläufe aufzubrechen und durch neue Handlungs- und Verhaltensweisen zu ersetzen. Dazu werden konkrete Hinweise zum Stromsparen und haushaltsindividuelle und -spezifische Verhaltens- und Prozessalternativen angeboten. Beide Komponenten werden auf der Basis von fünf mehr oder weniger aufeinander aufbauenden Modulen umgesetzt.

Über das Modul 1 wird auf der Haushaltsebene eine statische Analyse des aggregierten Gesamtverbrauchs des Haushaltes vorgenommen, wozu die Software dem Verbraucher einen historischen Vergleich des eigenen Verbrauchs über die Zeit und einen Referenzvergleich mit dem Durchschnittsverbrauch sozialstrukturell ähnlicher Haushalte ermöglicht. Beim Modul 2 wird der Gesamtlastgang in höherer Auflösung visualisiert. Für beide Module werden sowohl absolute Verbrauchswerte und monetäre Kosten als auch umweltspezifische Folgen des Verbrauchs ausgewiesen. Damit werden die „spezifischen Motive und die persönlichen und sozialen Normen“ (Fischer 2008) der jeweiligen Haushalte berücksichtigt.

Die Module 3 und 4 setzen auf der Geräteebene an. Mit dem Modul 3 sind gerätespezifische Verbrauchsanomalien das Ziel der Beratung, womit über die Umsetzung punktueller Maßnahmen beim Gerätepark, also Neuerwerb oder Reparatur, Einsparungen beim Stromverbrauch erzielt werden sollen. Die Maßnahmen erfordern keine Eingriffe in Haushaltsroutinen und -prozesse. Das Modul 4 setzt sich mit der Nutzung der Geräte auseinander, wobei sowohl über aggregierte gerätespezifische Verbrauchswerte als auch über gerätespezifische Lastgänge ein Bewusstsein für den eigenen Verbrauch geschaffen werden soll. Dazu werden die Verbrauchsfolgen der Nutzung einzelner Geräte veranschaulicht und es werden allgemeine Nutzungsempfehlungen für einen sparsameren Umgang gegeben.

Abbildung 2: Überblick über die fünf Module des Beratungssystems

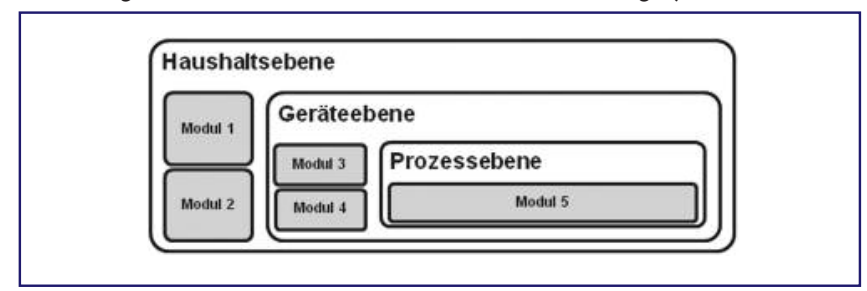

Quelle: Eigene Darstellung

\section{„Es können Empfehlungen für eine höhere Effizienz ohne Komfortverlust gegeben werden."}

Das Modul 5 nimmt auf der Ebene der im Haushalt ablaufenden Prozesse die detaillierteste Beratungsleistung wahr und unternimmt den Versuch der Umsetzung der Optimierung der stromverbrauchenden Haushaltsprozesse. Während zuvor die Gerätenutzung noch isoliert von den im Haushalt ablaufenden Haushaltsroutinen und -prozessen betrachtet wird, wird sich nun auf die Prozessebene konzentriert. Das Modul stellt damit aus doppelter Hinsicht den anspruchsvollsten Teil des Beratungssystems dar. Auf der einen Seite ist der Anspruch an das System besonders hoch, da sowohl die Erkennung der Haushaltsprozesse als auch die Beratung hinsichtlich der Optimierung dieser Prozesse ein hohes Maß an methodischer Komplexität aufweist. Auf der anderen Seite stellt das Modul aber auch höchste Ansprüche an den Verbraucher, da es nun um das Hinterfragen von über einen langen Zeitraum hinweg etablierten Haushaltsroutinen geht. Dabei lassen sich die Vorschläge und Optimierungsempfehlungen des Beratungssystems nach unterschiedlichen Schwierigkeits- beziehungsweise Intensitätsgraden unterscheiden, sodass der Nutzer selbst wählen kann, wie einschneidend die ihm vorgeschlagenen Verhaltens- und Prozessalternativen möglicherweise sind. Es lassen sich Empfehlungen mit dem Ziel einer höheren Effizienz (Veränderungen der Prozessorganisation ohne einen Komfortverlust oder anderweitige grundlegende Einbußen in der Haushaltsproduktion) von Empfehlungen mit dem Ziel der Suffizienz (Infragestellen des intendierten Nutzens) unterscheiden.

\section{Datenschutzrechtliche Bewertung}

Eine softwaregestützte Energieberatung kann für Energiekunden viele Vorteile bringen, birgt allerdings auch einige datenschutzrechtliche Risiken. Mit dem Einsatz einer solchen Energieberatung liegen erstmals Echtzeitinformationen zum Energieverbrauch von Privatpersonen vor, die es ohne das System und Sub- beziehunsgweise Smart-Metering nicht geben würde. Die Auswertung der Verbrauchswerte lässt Aussagen über persönliches Verhalten und Lebensgewohnheiten der Betroffenen zu (4). Diese Auswertung der Verbrauchsdaten ist nicht nur für den Betroffenen im Rahmen der Energieberatung interessant, es ist denkbar, dass Begehrlichkeiten von Dritten geweckt werden. Die Verarbeitung personenbezogener Daten ist in Deutschland durch das Bundesdatenschutzgesetz (BDSG) geschützt. Gemäß dem BDSG ist eine Erhebung, Verarbeitung oder Nutzung personenbezogener Daten unzulässig, es sei denn es liegt eine Erlaubnisnorm, eine Einwilligung des Betroffenen oder ein höherwertiges Gesetz, also ein Verbot mit Erlaubnisvorbehalt, vor. Gemäß der Zweckgebundenheit sind eine Ver- $\rightarrow$ 
wendung der im Rahmen der Energieberatung erhobenen Messwerte und deren Auswertung für andere Zwecke nur so weit zulässig, als die Einwilligung des Betroffenen es zulässt. Die erfassten Verbrauchswerte sind hochaufgelöst. In welcher Auflösung sie für eine effektive Energieberatung notwendig sind, kann derzeit noch nicht beantwortet werden. Es ist daher rückblickend zu prüfen, ob auf Daten im Rahmen der Energieberatung verzichtet werden kann.

Da es sich um sensible Informationen handelt, die im Rahmen der Energieberatung erfasst werden, wird im Rahmen dieses Ansatzes der Datenschutz bereits bei der Konzeption der Energieberatung mitberücksichtigt. Der hier vorgestellte Beratungsansatz verzichtet bewusst auf eine Weblösung, die Verbrauchsdaten online speichert wie zum Beispiel Google PowerMeter (5). Die Energieberatung wird als lokales Desktopprogramm bei den Betroffenen installiert. Da es keinen personellen Berater gibt und der Betroffene direkt mit der Energieberatungssoftware selbst arbeiten kann, besitzt der Betroffene auch die volle Kontrolle über seine Daten.

\section{Ausblick}

Im Rahmen des Projekts eTelligence wird der hier skizzierte Beratungsansatz durch zwei Software-Prototypen ansatzweise realisiert und mit einer kleinen Anzahl von Test-Nutzern evaluiert werden. Hierdurch soll im Wesentlichen ein "proof of concept" erfolgen, der auch Auskunft über die Anforderungen an die notwendigen Smart-Metering-Techniken und die Akzeptanz bei den Nutzern geben soll. Es ist zu erwarten, dass sowohl bezüglich der automatischen Prozesserkennung als auch bezüglich der Breite der automatisierten Beratung weitere Arbeiten folgen müssen, um diesen neuen Ansatz zur Energieberatung in eine breite Nutzung zu überführen.

\section{Anmerkungen}

(1) http://www.e-energy.de/de/etelligence.php - Teilprojekt 3.4

(2) http://stromsparcheck.stromeffizienz.de/

(3) http://www. $\mathrm{CO}_{2}$ online.de/kampagnen-und-projekte/energiespar-ratgeber/

(4) http://www.heise.de/security/meldung/Intelligente-Stromnetze-Ich-weissob-du-gestern-geduscht-hast-864221.htm

(5) http://www.google.com/powermeter

\section{Literatur}

Arvola, A. / Uutela, A. / Anttila, U.: Billing feedback as means to encourage household electricity conservation: A field experiment in Helsinki. Paper presented at the The Energy Efficiency Challange for Europe, Rungstedgard, Denmark 1993.

Baranski, M.: Energie-Monitoring im privaten Haushalt. Dissertation, Universität Paderborn, Paderborn 2006.

Bitsch, R. / Feldmann, W. / Aumayer, G.: Virtuelle Kraftwerke - Einbindung dezentraler Energieerzeugungsanlagen. In: etz 9/2002.

Brohmann, B. / Cames, M. / Herold, A.: Klimaschutz durch Minderung von Treibhausgasemissionen im Bereich Haushalte und Kleinverbrauch durch klimagerechtes Verhalten. Band 1: Private Haushalte. Freiburg, Darmstadt, Berlin 2000.

Bürger, V.: Quantifizierung und Systematisierung der technischen und verhaltensbedingten Stromeinsparpotentiale der deutschen Privathaushalte. In: Zeitschrift für Energiewirtschaft 34, 2/2010, S. 47-59.
Dahlstrand, U. / Biehl, A.: Pro-Environmental Habits: Propensity Levels in Behavioral Change. In: Journal of Applied Social Psychology 27, 1997, S. 588601.

Darby, S.: The Effectiveness of Feedback on Energy Consumption. A review for DEFRA of the Literature on Metering, Billing and Direct Displays. Environmental Change Institute, University of Oxford 2006.

Deutsche Energie-Agentur: Integration Erneuerbarer Energien in die Deutsche Stromversorgung im Zeitraum 2015 - 2020 mit Ausblick auf 2025. Berlin 2010.

Duscha, M. / Dünnhoff, E. / Ivanov, M. / Wegener, S.: Effiziente Beratungsbausteine zur Verminderung des Stromverbrauchs in privaten Haushalten. ifeu - Institut für Energie- und Umweltforschung Heidelberg GmbH. Heidelberg 2006.

Fischer, C.: Feedback on household electricity consumption: a tool for saving energy? Energy Efficiency 1/2008, S. 79-104.

Hart, G.: Nonintrusive Appliance Load Monitoring. In: Proceeding of the IEEE 80, 1992, S. 1870-1891.

Jahn, Th.: Sozial-ökologische Forschung. Ein neuer Forschungstyp in der Nachhaltigkeitsforschung. In: Linne, G. / Schwarz, M. (Hg.): Handbuch Nachhaltige Entwicklung. Wie ist nachhaltiges Wirtschaften machbar? Opladen 2003, S. 545-555.

Klingenberg, T.: Smart Submetering - Effizienter Einsatz von Submetern zur Aktivitätsbestimmung in Privathaushalten mit Hilfe adaptiver Lernverfahren. Diplomarbeit in Informatik, Universität Oldenburg, Oldenburg 2010.

Pihala, H.: Non-intrusive appliance load monitoring system based on a modern kWh-Meter. In: VTT Publications. 1998.

Schleich, J. / Klobasa, M. / Brunner, M. / Gölz, S. / Götz, K. / Sunderer, G.: Smart Metering in Germany and Austria - results of providing feedback information in a field trial. Working Paper Sustainability and Innovation $6 / 2011$.

Sonnenschein, M. / Rapp, B. / Bremer, J.: Demand Side Management und Demand Response. In: Beck, H.-P. / Buddenberg, J. / Meller, E. / Salander C. (Hrsg.): Handbuch Energiemangement. 2010.

Ueno, T. / Sano, F. / Saeki, O. / Tsuji, K.: Effectiveness of an energy-consumption information system on energy savings in residential houses based on monitored data. In: Applied Energy 83, 2006, S. 166-183.

\section{AUTOREN + KONTAKT}

Dr. Michael Sonnenschein ist Professor an und Leiter der Abteilung Umweltinformatik im Department für Informatik der Universität Oldenburg und Bereichsvorstand „Energie" im OFFIS Institut für Informatik.

E-Mail: sonnenschein@offis.de

Dr. Thorsten Raabe ist Professor und Inhaber des Lehrstuhls für allgemeine Betriebswirtschaftslehre und Marketing an der Carl von Ossietzky Universität Oldenburg.

E-Mail: thorsten.raabe@uni-oldenburg.de

Dr. Petra Beenken ist wissenschaftliche Mitarbeiterin, Christopher-Marcel Meinecke und Dipl.-Inform. Andreas Hüwel sind wissenschaftliche Mitarbeiter im OFFIS Institut für Informatik in Oldenburg.
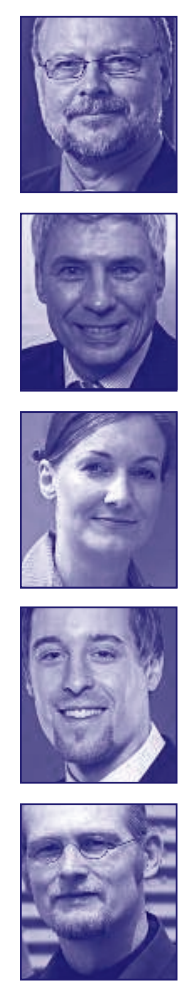


\section{Lizenzhinweis}

Die Beiträge in ÖkologischesWirtschaften werden unter der Creative-Commons-Lizenz "CC 4.0 Attribution Non-Commercial No Derivatives" veröffentlicht. Im Rahmen dieser Lizenz muss der Autor/Urheber stets genannt werden, das Werk darf nicht bearbeitet, abgewandelt oder in anderer Weise verändert und außerdem nicht kommerziell genutzt werden. Die digitale Version des Artikels bleibt für zwei Jahre Abonnent/innen vorbehalten und ist danach im Open Access verfügbar. 